\title{
Article \\ Simple Analytical Expression of the Voigt Profile
}

\author{
Salma Chib $(1)$ and Abdelmajid Belafhal *
}

check for

updates

Citation: Chib, S.; Belafhal, A. Simple Analytical Expression of the Voigt Profile. Quantum Rep. 2022, 4, 36-46. https://doi.org/10.3390/ quantum4010004

Academic Editor: Gerald B. Cleaver

Received: 25 November 2021

Accepted: 25 January 2022

Published: 28 January 2022

Publisher's Note: MDPI stays neutral with regard to jurisdictional claims in published maps and institutional affiliations.

Copyright: (C) 2022 by the authors. Licensee MDPI, Basel, Switzerland. This article is an open access article distributed under the terms and conditions of the Creative Commons Attribution (CC BY) license (https:// creativecommons.org/licenses/by/ $4.0 /)$.

\author{
Laboratory LPNAMME, Laser Physics Group, Department of Physics, Faculty of Sciences, \\ Chouaib Doukkali University, P.B 20, El Jadida 24000, Morocco; salmachib@gmail.com \\ * Correspondence: belafhal@gmail.com
}

\begin{abstract}
This work examines several analytical evaluations of the Voigt profile, which is a convolution of the Gaussian and Lorentzian profiles, theoretically and numerically. Mathematical derivations are performed concisely to illustrate some closed forms of the considered profile. A representation in terms of special function and a simple and interesting approximation of the Voigt function are well demonstrated, which could have promising applications in several fields of physics, e.g., atmospheric radiative transfer, neutron reactions, molecular spectroscopy, plasma waves, and astrophysical spectroscopy.
\end{abstract}

Keywords: Voigt profile; Gaussian profile; Lorentzian profile; special functions

\section{Introduction}

Special interest has been paid since the beginning of the last century to the Voigt function introduced in the literature by Reiche [1] and emerged intensively in a wide variety of fields of physics due to their novel features, such as infrared lines [2], astrophysics [3], normal atmosphere and the spectrum line profiles [4-6], and astrophysical spectroscopy [7]. In this last field, the Voigt function plays an important role such as to evaluate the opacities of hot stellar gases. In spectral line theory, the combined Doppler and Lorentz broadening of the spectral line is described by the Voigt function because the absorption coefficient of a gas is proportional to the considered function.

As is known, many computational procedures used to evaluate the Voigt function are published [8-17]. On the other hand, Srivastava and Miller have investigated a unified study of the Voigt functions [18]. In fact, the difficulty of programming the exact expression of the Voigt function prevents the advancement of certain studies in physics. Motivated by this reason, in this work, the authors provide researchers with a simple and easy-to-handle approximation in terms of some special functions of one and two variables.

In 1995, we used this profile to treat about 400 absolute measured intensities of Carbon Oxysulfide recorded in the range between 2500 and $3100 \mathrm{~cm}^{-1}$ using a Bruker IFS120HR spectrometer [19]. Additionally, we investigated [20] the shape of spectral lines given by the Voigt profile by establishing a numerical evaluation of the integrated absorption of a spectral line with the considered profile. Earlier, AlOmar [21] presented some Chebyshev approximations for the spectral features of the Voigt profile.

In the present study, we will derive several representations of the Voigt profile $V(x, y)$ defined by

$$
V(x, y)=\frac{1}{\alpha_{D}}\left(\frac{\ln 2}{\pi}\right)^{1 / 2} K(x, y)
$$

where

$$
K(x, y)=\frac{y}{\pi} \int_{-\infty}^{+\infty} \frac{e^{-t^{2}}}{y^{2}+(x-t)^{2}} d t
$$


is the Voigt function or the Voigt profile function, with

$$
x=\frac{\left(v-v_{0}\right)}{\alpha_{D}}(\ln 2)^{1 / 2},
$$

and

$$
y=\frac{\alpha_{L}}{\alpha_{D}}(\ln 2)^{1 / 2} .
$$

These last quantities are called the wavenumber scale in units of Doppler width and the ratio of Lorentz to Doppler widths, respectively. In these last equations, $\alpha_{D}$ and $\alpha_{L}$ are the Doppler half-width and the Lorentz half-width; $v_{0}$ is the wave number at the line center, and $v$ is the wave number at which the Voigt function is to be evaluated.

The results of the present investigation will be given in terms of some series of familiar special functions of mathematical physics. This paper is organized as follows: in Section 2, the evolution of the Voigt profile in terms of the Humbert function, and as a simple approximation, is given. Of our main results, numerical discussions are performed in Section 3. Finally, the conclusion of our work is presented in Section 4.

\section{Evaluation of the Voigt Profile}

\subsection{Expression of $V$ in Terms of the Humbert Function}

The Voigt function can be expressed in the general form as follows

$$
K_{\mu, v}(p, q, \chi)=\sqrt{\frac{\chi}{2}} \int_{0}^{+\infty} \rho^{\mu} e^{-p \rho^{2}+2 q \rho} J_{v}(\chi \rho) d \rho .
$$

To compute the generalized Voigt function $K_{\mu, \nu}(p, q, \chi)$, we first evaluate the following integral

$$
\int_{0}^{+\infty} \rho^{\mu} J_{v}(\chi \rho) e^{-p \rho^{2}+2 q \rho} d \rho, \text { with } \operatorname{Re}(p)>0 \text { and } \operatorname{Re}(\mu)>-1 .
$$

Using the identity $e^{x}=\operatorname{ch} x+\operatorname{sh} x$ and the following series representation of the Bessel function [22]

$$
J_{v}(z)=\left(\frac{z}{2}\right)^{v} \sum_{k=0}^{+\infty} \frac{\left(-z^{2} / 4\right)^{k}}{k !} \frac{1}{\Gamma(v+1+k)} ; \forall z \in \mathbb{C} \backslash(-\infty, 0),
$$

one finds from Equation (5)

$$
K_{\mu, v}(p, q, \chi)=\left(\frac{\chi}{2}\right)^{v+\frac{1}{2}} \sum_{k=0}^{+\infty} \frac{\left(-\chi^{2} / 4\right)^{k}}{k ! \Gamma(v+1+k)} I_{k}
$$

where

$$
I_{k}=\int_{0}^{\infty} \rho^{\mu+2 k+v} \exp \left(-p \rho^{2}\right) \operatorname{ch}(2 q \rho) d \rho+\int_{0}^{\infty} \rho^{\mu+2 k+v} \exp \left(-p \rho^{2}\right) \operatorname{sh}(2 q \rho) d \rho .
$$

With the help of the following identities [23]

$$
\int_{0}^{\infty} x^{2 \alpha-1} \exp \left(-\beta x^{2}\right) \operatorname{ch}(\gamma x) d x=\frac{\Gamma(2 \alpha)}{2}(2 \beta)^{-\alpha} \exp \left(\frac{\gamma^{2}}{8 \beta}\right)\left[D_{-2 \alpha}\left(-\frac{\gamma}{\sqrt{2 \beta}}\right)+D_{-2 \alpha}\left(\frac{\gamma}{\sqrt{2 \beta}}\right)\right],
$$


and

$$
\int_{0}^{\infty} x^{2 \alpha-1} \exp \left(-\beta x^{2}\right) \operatorname{sh}(\gamma x) d x=\frac{\Gamma(2 \alpha)}{2}(2 \beta)^{-\alpha} \exp \left(\frac{\gamma^{2}}{8 \beta}\right)\left[D_{-2 \alpha}\left(-\frac{\gamma}{\sqrt{2 \beta}}\right)-D_{-2 \alpha}\left(\frac{\gamma}{\sqrt{2 \beta}}\right)\right],
$$

with $\operatorname{Re}(\alpha)>0, \operatorname{Re}(\beta)>0$ and $D_{-2 \alpha}$ being the parabolic cylinder function given in terms of the Kummer's function ${ }_{1} F_{1}$ as

$$
D_{-2 \alpha}(z)=\frac{\sqrt{\pi}}{2^{\alpha}} \exp \left(-z^{2} / 4\right)\left\{\frac{1}{\Gamma\left(\alpha+\frac{1}{2}\right)} 1 F_{1}\left(\alpha ; \frac{1}{2} ; \frac{z^{2}}{2}\right)-\frac{\sqrt{2}}{\Gamma(\alpha)} z_{1} F_{1}\left(\alpha+\frac{1}{2} ; \frac{3}{2} ; \frac{z^{2}}{2}\right)\right\},
$$

and after some algebraic manipulations, one obtains the following expression of Equation (5)

$$
\begin{aligned}
K_{\mu, v}(p, q, \chi) & =\sqrt{\frac{\chi}{2}} \frac{\sqrt{\pi}}{(4 p)^{\frac{\mu+1}{2}}}\left(\frac{\chi}{4 \sqrt{p}}\right)^{v}\left[\sum_{k=0}^{+\infty} \frac{\left(-\chi^{2} / 16 p\right)^{k}}{k ! \Gamma(v+1+k)} \eta_{1, k 1} F_{1}\left(\frac{\mu+v+1}{2}+k ; \frac{1}{2} ; \frac{q^{2}}{p}\right)\right. \\
& \left.+\frac{2 q}{\sqrt{p}} \sum_{k=0}^{+\infty} \frac{\left(-\chi^{2} / 16 p\right)^{k}}{k ! \Gamma(v+1+k)} \eta_{2, k 1} F_{1}\left(\frac{\mu+v}{2}+1+k ; \frac{3}{2} ; \frac{q^{2}}{p}\right)\right],
\end{aligned}
$$

where

$$
\eta_{1, k}=\frac{\Gamma(\mu+v+1+2 k)}{\Gamma\left(\frac{\mu+v}{2}+1+k\right)}
$$

and

$$
\eta_{2, k}=\frac{\Gamma(\mu+v+1+2 k)}{\Gamma\left(\frac{\mu+v+1}{2}+k\right)} .
$$

Making use of the above equations and the two following identities [24]

$$
(\lambda+m)_{n}=\frac{(\lambda)_{m+n}}{(\lambda)_{m}}
$$

and

$$
\Gamma(2 z)=\frac{2^{2 z-1}}{\sqrt{\pi}} \Gamma(z) \Gamma\left(z+\frac{1}{2}\right) \text { with } z \neq 0,-\frac{1}{2},-1,-\frac{3}{2}, \ldots,
$$

Equation (12) can be written in the following form

$$
\begin{gathered}
K_{\mu, v}(p, q, \chi)=\sqrt{\frac{\chi}{2}} \frac{1}{2 p^{\frac{\mu+1}{2}}} \frac{(\chi / 2 \sqrt{p})^{v}}{v !}\left\{\Gamma\left(\frac{\mu+v+1}{2}\right) \Psi_{2}\left[\frac{\mu+v+1}{2} ; v+1, \frac{1}{2} ;-\frac{\chi^{2}}{4 p}, \frac{q^{2}}{p}\right]\right. \\
\left.+\frac{2 q}{\sqrt{p}} \Gamma\left(\frac{\mu+v}{2}+1\right) \Psi_{2}\left[\frac{\mu+v}{2}+1 ; v+1, \frac{3}{2} ;-\frac{\chi^{2}}{4 p}, \frac{q^{2}}{p}\right]\right\},
\end{gathered}
$$

where $\Psi_{2}$ is the Humbert function given by

$$
\Psi_{2}\left[\alpha ; \gamma, \gamma^{\prime} ; x, y\right]=\sum_{m, n=0}^{\infty} \frac{(\alpha)_{m+n}}{(\gamma)_{m}\left(\gamma^{\prime}\right)_{n}} \frac{x^{m}}{m !} \frac{y^{n}}{n !},
$$

with $|x|<\infty$ and $|y|<\infty$.

In the case of $\chi=x$ and $q=-\frac{y}{2}$, Equation (18) can be written as

$$
\begin{aligned}
K_{\mu, v}(p, x, y) & =\sqrt{\frac{x}{2}} \int_{0}^{\infty} t^{\mu} \exp \left(-p t^{2}-y t\right) J_{v}(x t) d t \\
& =\sqrt{\frac{x}{2}} \frac{1}{2 p^{\frac{\mu+1}{2}}} \frac{(x / 2 \sqrt{p})^{v}}{v !}\left\{\Gamma(\sigma) \Psi_{2}\left[\sigma ; v+1, \frac{1}{2} ;-\frac{x^{2}}{4 p}, \frac{y^{2}}{4 p}\right]\right. \\
& \left.-\frac{2 y}{\sqrt{p}} \Gamma\left(\sigma+\frac{1}{2}\right) \Psi_{2}\left[\sigma+\frac{1}{2} ; v+1, \frac{3}{2} ;-\frac{x^{2}}{4 p}, \frac{y^{2}}{4 p}\right]\right\},
\end{aligned}
$$


where

$$
\sigma=\frac{\mu+v+1}{2}
$$

Equation (20) is a generalization of the Voigt function $K_{\mu, v}(p, x, y)$ involving the Humbert function. This equation represents our first main result in the current study.

By taking $p=\frac{1}{4}$, Equation (20) becomes

$$
\begin{aligned}
& K_{\mu, v}\left(\frac{1}{4}, x, y\right)=K_{\mu, v}(x, y)=\sqrt{\frac{x}{2}} \int_{0}^{\infty} t^{\mu} \exp \left(-y t-\frac{t^{2}}{4}\right) J_{v}(x t) d t \\
& \quad=\sqrt{\frac{x}{2}} \frac{2^{\mu} x^{v}}{v !}\left\{\Gamma(\sigma) \Psi_{2}\left[\sigma ; v+1, \frac{1}{2} ;-x^{2}, y^{2}\right]-2 y \Gamma\left(\sigma+\frac{1}{2}\right) \Psi_{2}\left[\sigma+\frac{1}{2} ; v+1, \frac{3}{2} ;-x^{2}, y^{2}\right]\right\} .
\end{aligned}
$$

This particular case is the result published in Refs. [18,25].

In the following, we will use the case of $v=-1 / 2$. We know that $J_{-\frac{1}{2}}(z)=\sqrt{\frac{2}{\pi z}} \cos z$ and Equation (22) can be expressed, in this case, as

$$
K_{\mu,-\frac{1}{2}}(x, y)=\frac{1}{\sqrt{\pi}} \int_{0}^{\infty} t^{\mu-\frac{1}{2}} \exp \left(-y t-\frac{t^{2}}{4}\right) \cos (x t) d t
$$

which yields, for $\mu=1 / 2$, the formula

$$
K_{\frac{1}{2},-\frac{1}{2}}(x, y)=\left\{\Psi_{2}\left[\frac{1}{2} ; \frac{1}{2}, \frac{1}{2} ;-x^{2}, y^{2}\right]-\frac{2 y}{\sqrt{\pi}} \Psi_{2}\left[1 ; \frac{1}{2}, \frac{3}{2} ;-x^{2}, y^{2}\right]\right\} .
$$

Finally, the Voigt profile is obtained as

$$
V(x, y)=\frac{1}{\pi \omega_{G}}\left\{\sqrt{\pi} \Psi_{2}\left[\frac{1}{2} ; \frac{1}{2}, \frac{1}{2} ;-x^{2}, y^{2}\right]-2 y \Psi_{2}\left[1 ; \frac{1}{2}, \frac{3}{2} ;-x^{2}, y^{2}\right]\right\},
$$

with $\omega_{G}$ being the Gaussian width.

\subsection{A Simple Approximation of the Voigt Profile}

In 2011, Huang et al. [26] studied some laser beams' diffraction phenomena by an opaque disk. This application, used in optics and astrophysics domains, yields the use of a particular integral that is similar to the Voigt profile with a difference in the integrand with some modification involving a product of the Bessel function of zero order. This integral given in Ref. [26] is defined by

$$
T^{(\alpha)}(x, y)=\int_{-\infty}^{+\infty} \frac{\Delta \omega}{1+\tau^{2}\left(\omega_{0}+\Delta \omega t\right)^{2}} \exp \left(-t^{2}\right) J_{0}^{2}\left(\alpha\left(\omega_{0}+\Delta \omega t\right)\right) d t,
$$

where $\alpha \geq 0$

By taking $y=\frac{1}{\tau \Delta \omega}$ and $x=-\frac{\omega_{0}}{\Delta \omega} \rightarrow \Delta \omega=-\frac{\omega_{0}}{x}$, so $\tau \Delta \omega=\frac{1}{y}$. Then, $1+\tau^{2} \Delta \omega^{2}\left(\frac{\omega_{0}}{\Delta \omega}+t\right)^{2}$ $=1+\left(\frac{x-t}{y}\right)^{2}$.

Finally, the quantity $T^{(\alpha)}(x, y)$ can be rewritten in the following form

$$
T^{(\alpha)}(x, y)=\int_{-\infty}^{+\infty} \frac{1}{1+\left(\frac{x-t}{y}\right)^{2}} \exp \left(-t^{2}\right) J_{0}^{2}\left[\alpha\left(1-\frac{t}{x}\right)\right] d t
$$

For $\alpha=0$, the last quantity can be performed by using theorem 2 of Ref. [27], but for simplicity, we will follow the procedure below. 
Note that this integral cannot be evaluated analytically but one can use a Taylor-type limited development versus the parameter $1 / x$ of the integrand. For that, we will use the fourth-order development and obtain the following expression

$$
\begin{aligned}
T^{(\alpha)}(x, y) & \approx \frac{\sqrt{\pi}}{C(x, y)}\left\{J_{0}^{2}(\alpha)-\frac{1}{2 y^{2} C^{2}(x, y)}\left[1-\frac{3 x^{2}}{y^{2}}+\frac{\alpha^{2} y^{2}}{x^{2}} C^{2}(x, y)\right] J_{0}^{2}(\alpha)\right. \\
& \left.+\frac{1}{2 x^{2} C^{2}(x, y)}\left[\alpha\left(1+\frac{6 x^{2}}{y^{2}}+\frac{5 x^{4}}{y^{4}}\right) J_{0}(\alpha) J_{1}(\alpha)+\alpha^{2} C^{2}(x, y) J_{1}^{2}(\alpha)\right]\right\} O\left(\frac{1}{x}\right)^{4}
\end{aligned}
$$

where

$$
C(x, y)=1+\frac{x^{2}}{y^{2}}
$$

Taking $\alpha=0$, so $J_{0}(\alpha)=1$ and $J_{1}(\alpha)=0$, and consequently Equation (27) becomes

$$
T^{(0)}(x, y)=T(x, y) \approx \frac{\sqrt{\pi}}{C(x, y)}\left[1-\frac{1}{2 y^{2} C^{2}(x, y)}\left(1-\frac{3 x^{2}}{y^{2}}\right)\right]+O\left(\frac{1}{x}\right)^{4} .
$$

Accordingly, the Voigt profile may be written as

$$
V(x, y)=\frac{1}{\alpha_{D}} \sqrt{\frac{\ln 2}{\pi}} K(x, y)
$$

where

$$
K(x, y)=\frac{1}{\pi y} T(x, y) \approx \frac{1}{\sqrt{\pi} y C(x, y)}\left[1-\frac{1}{2 y^{2} C^{2}(x, y)}\left(1-\frac{3 x^{2}}{y^{2}}\right)\right]+O\left(\frac{1}{x}\right)^{4} .
$$

This last equation is our second main result obtained in the present investigation and expresses the simple formula that describes the Voigt function.

Equation (30) corresponds to the Voigt profile and is valid only for an important region of the parameters $x$ and $y$, which we will discuss in the next Section and compare to our evaluation [20] given by

$$
V(x, y)=\frac{S}{\pi \alpha_{D}}\left(\frac{\ln 2}{\pi}\right)^{\frac{1}{2}} \pi K(x, y)
$$

where

$$
\begin{aligned}
K(x, y)= & \operatorname{Re}\left\{\operatorname { e x p } ( - z ^ { 2 } - y ^ { 2 } ) \left[k_{1} t+k_{2} t^{2}+k_{3} t^{3}+k_{4} t^{4}+k_{5} t^{5}-\frac{1}{2 \pi y}[(1-\cos 2 x y-i \sin 2 x y)]\right.\right. \\
& \left.\left.-\frac{2}{\pi} \sum_{n=1}^{\infty} \frac{\exp \left(-n^{2} / 4\right)}{n^{2}+4 y^{2}}\left[f_{n}(y,-x)+i g_{n}(y,-x)\right]\right]\right\},
\end{aligned}
$$

with

$$
\begin{gathered}
z=x+i y, k_{1}=0.254829592, k_{2}=-0.284496736, k_{3}=1.421413741, k_{4}=-1.453152027, k_{5}=1.061405429 \\
p=0.3275911, t=\frac{1}{(1+p y)}, f_{n}(y,-x)=2 y-2 y \cosh (-n x) \cos (2 x y)-n \sinh (-n x) \sin (2 x y)
\end{gathered}
$$

and

$$
g_{n}(y,-x)=-2 y \cosh (-n x) \sin (2 x y)+n \sinh (-n x) \cos (2 x y) .
$$

Our focal point of the above Subpart is to present a simple formula that describes a Voigt profile and indeed we have arrived at our main objective that is expressed by Equation (31).

\section{Analysis and Numerical Discussion}

In the preceding Section, several expressions to approximate the Voigt function are developed after tedious algebraic manipulations (see Equations (20) and (31)). In the first step, a comparison between our simple approximation (Equation (31)) and that of Teodorescu et al. [13] with the exact expression presented by Srivastava [18] is given. Afterwards, the evolution of the generalized expression of the Voigt function given by Equation (20) will be studied. Suitable parameters are chosen to perform it. Then, due to 
its accuracy and its fast compilation time, the simple approximation of the Voigt function $K(x, y)$ as a function of $x$ and $y$, which is expressed by Equation (31) will be simulated. To demonstrate the validity of our simple approach and the interval of its application, graphical representations are used to compare it to our previous result (Equation (33)) and the numerical integration of Equation (2). The numerical method used to compute the integral form of the Voigt function is explained in detail in the following paragraph.

According to Gauss-Hermite quadrature, the integral cited in Equation (2) can be rewritten in the below form

$$
K(x, y)=\frac{y}{\pi} \int_{-\infty}^{+\infty} \frac{e^{-t^{2}}}{y^{2}+(x-t)^{2}} d t=\frac{y}{\pi} \sum_{i=1}^{n} w_{i} f\left(t_{i}\right),
$$

where $f\left(t_{i}\right)=\frac{1}{y^{2}+\left(x-t_{i}\right)^{2}}$, with $w_{i}$ is the weight factors and $t_{i}$ is the abscissas which are illustrated in Table 1. For more accuracy, we take $n=20$.

Table 1. Table of abscissas and weight factors for Gauss-Hermite integration.

\begin{tabular}{ccc}
\hline $\boldsymbol{i}$ & Abscissas $= \pm \boldsymbol{t}_{\boldsymbol{i}}$ & $\boldsymbol{w}_{\boldsymbol{i}}$ \\
\hline 1 & 0.2453407083009 & $(-1) 4.622436696006$ \\
2 & 0.7374737285454 & $(-1) 2.866755053628$ \\
3 & 1.2340762153953 & $(-1) 1.090172060200$ \\
4 & 1.7385377121166 & $(-2) 2.481052088746$ \\
5 & 2.2549740020893 & $(-3) 3.243773342238$ \\
6 & 2.7888060584281 & $(-4) 2.283386360163$ \\
7 & 3.3478545673832 & $(-6) 7.802556478532$ \\
8 & 3.9447640401156 & $(-7) 1.086069370769$ \\
9 & 4.6036824495507 & $(-10) 4.399340992273$ \\
10 & 5.3874808900112 & $(-13) 2.229393645534$ \\
\hline
\end{tabular}

In the past decades, some studies have divided their fields of work into three regions to evaluate the Voigt profile function, where each region has its own specificity in terms of the method used in the treatment of the considered function. In this study, our work plan is composed of two computational regions and is characterized by two analytical expressions.

As an indication, the mathematical writing $(-1) 4.622436696006$ is equivalent to $4.622436696006 \times 10^{-1}$.

The two regions used to examine the evolution of the Voigt function are identified in Figure 1. This decomposition is made based on the simulation results.

Figure 2 illustrates a comparison between two approximations and an exact expression of the Voigt function. From Figure 2 we can observe that our simple approach expressed by Equation (31) fits the Voigt function very well with a relative average error of $5 \times 10^{-6}$ and a running time of $0.141 \mathrm{~s}$. On the other hand, the approximation of Teodorescu et al. requires a time of $0.161 \mathrm{~s}$ and a relative average error of $3 \times 10^{-5}$.

In Figure $3 a$, the properties of the generalized Voigt function versus $x$ with different values of $p$ were investigated, and in the same plot, the contour graph of a particular case of our obtained result was presented (see Figure $3 b$ ). The calculation parameters are set as $\mu=1 / 2, v=-1 / 2$ and $y=2.5$. According to Figure $3 a$, the profile of the generalized Voigt function maintains symmetry when $p$ varies. In addition, when $p$ is large, the width of the profile becomes more important. Furthermore, increasing the value of $p$ will decrease the maximum of the peak.

Figure 4 illustrates the evolution of the Voigt function in terms of $x$ using our old and new results as well as numerical integration. The difference between our old and new approaches with the numerical integration is plotted to test the degree of agreement. A disagreement between the two analytical expressions for $0.5 \leq y \leq 1.5$ and $x \leq 2$ is observed in Figure 4 . However, we can obtain $80 \%$ of agreement between our approaches and numerical evaluations from $y=2$ and for all values of $x$. Moreover, we can see that 
the Voigt profile function reaches its minimum values with the increase in the parameter $y$. On the other hand, we find that the Voigt profile function vanishes at infinity faster with a small value of $y$. The following conclusion can readily be drawn from Figure 4: Equation (33) correctly characterizes the Voigt function in region I. Note that the curves obtained in Figure $4 \mathrm{~d}$ are identical to the curve plotted in Figure $3 \mathrm{a}$ for $p=\frac{1}{4}$, which proves the equivalence between Equation (24) and Equations (31) and (33).

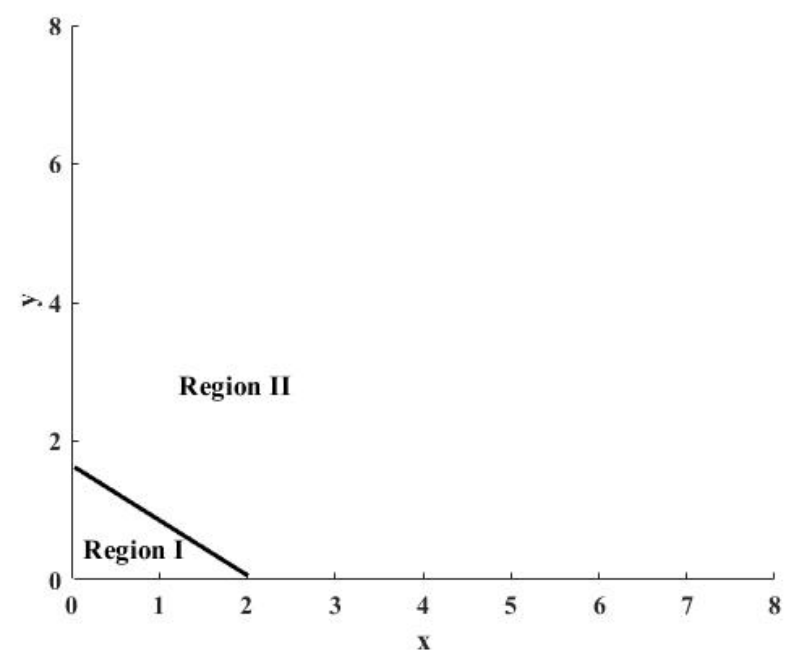

Figure 1. Different regions used in this paper.

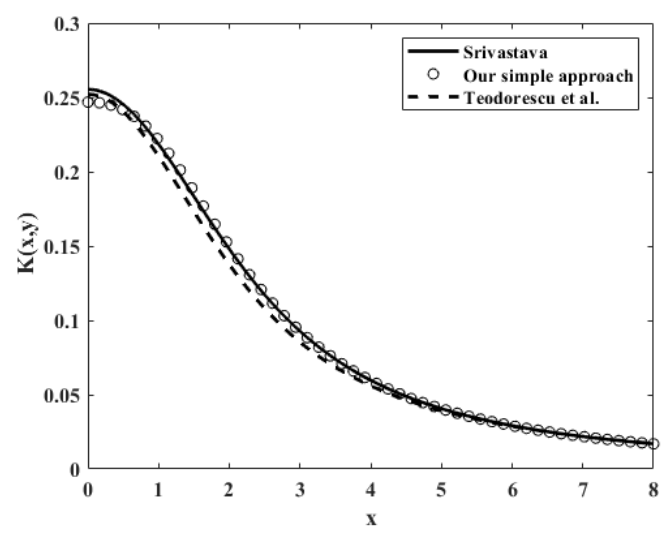

Figure 2. Illustration of the Voigt function $K(x, y)$ in terms of $x$ with $y=2$.

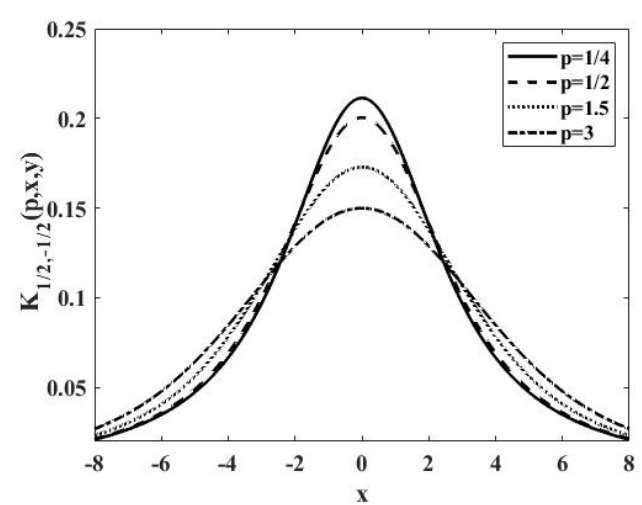

(a)

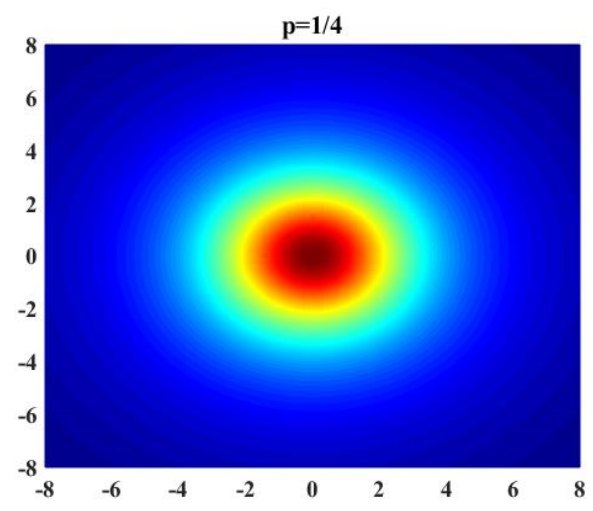

(b)

Figure 3. The generalized Voigt function $K_{1 / 2,-1 / 2}(p, x, y)$ distribution versus $x$ with (a) different values of $p$ and (b) contour graph for $p=1 / 4$. 

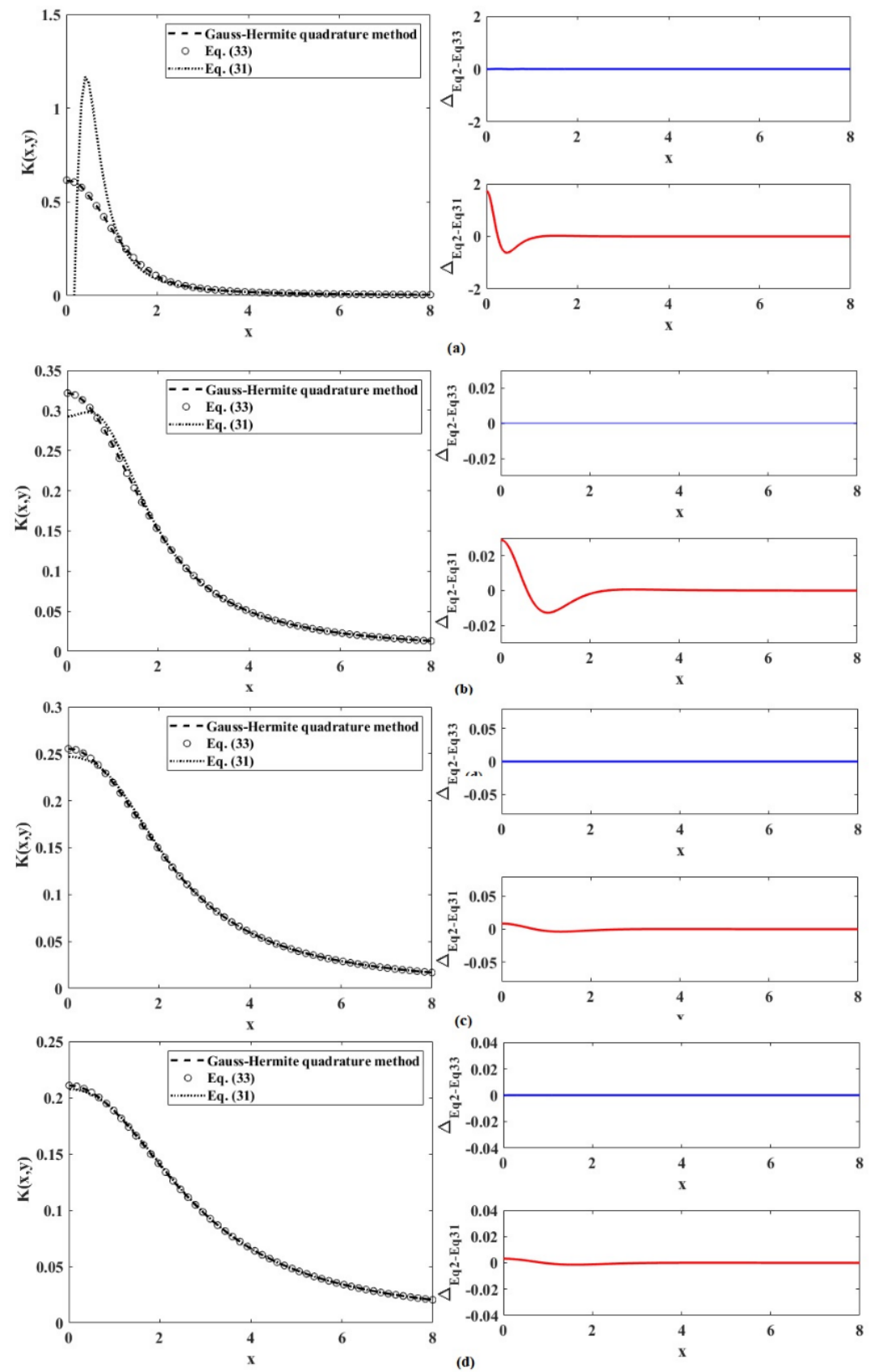

Figure 4. Illustration of the Voigt function $K(x, y)$ in terms of $x$ for different values of $y$ : (a) $y=0.5$, (b) $y=1.5$, (c) $y=2$, (d) $y=2.5$ and on the right side, the simulations of the difference between Equation (33), Equation (2) and Equation (31), Equation (2).

The variation of the Voigt function versus $x$ for several values of $y$ in the second region is plotted in Figure 5. It can observed that the Voigt function can be described by using the two simple approaches (Equations (31) and (33)). For a large value of $y(y \geq 6)$ and $0 \leq x \leq 2$, the old formula oscillates because it is expressed as a function of cosine and sine functions, whereas the new approximation remains valid for all values of $x$ and large values of $y$. Additionally, as the parameter $y$ is increased, the maximum of the peak decreases.

Some plots are depicted in Figure 6 to analyze the behavior of the Voigt function $K(x, y)$ as a function of $y$ and with several values of $x$. As shown in this figure, for a large value of $x$ the maximum of the peak reduces and the profile becomes wider.

Moreover, and by analyzing the comportment of this function from another viewpoint, we notice that for $x=2$ and small values of $y$ the curve of the new approach does not 
coincide with the two other curves that represent the old approximation and the numerical integration. Excellent compatibility between our analytical formulae and the numerical solution using the Gauss-Hermite quadrature method, is obtained from $x=3$ and for all values of $y$.
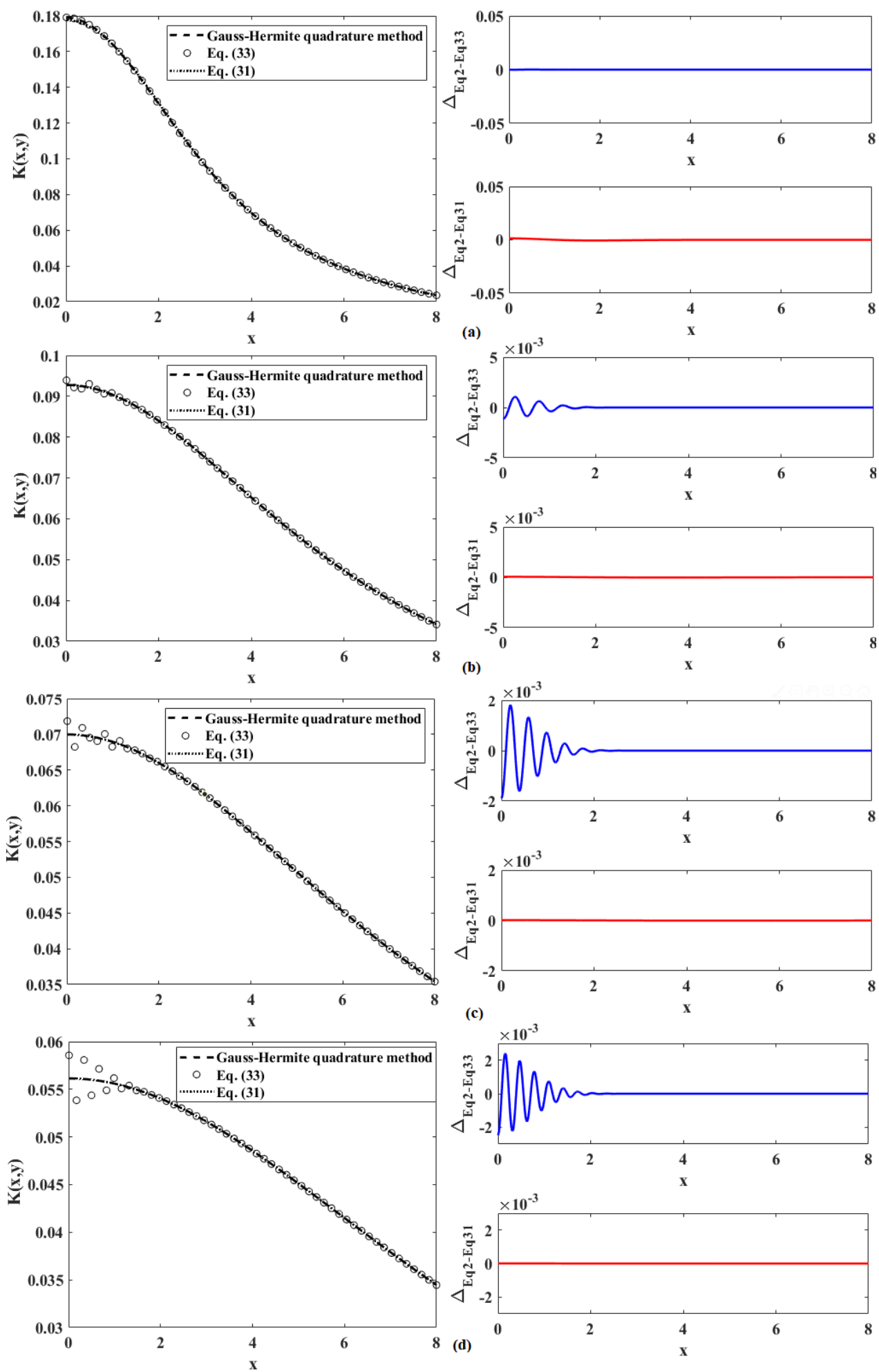

Figure 5. Evolution of the Voigt function $K(x, y)$ in terms of $x$ for different values of $y$ : (a) $y=3$, (b) $y=6$, (c) $y=8$ (d) $y=10$ and on the right side, the plots of the subtraction of the Gauss-Hermite result from the result of Equations (31) and (33). 

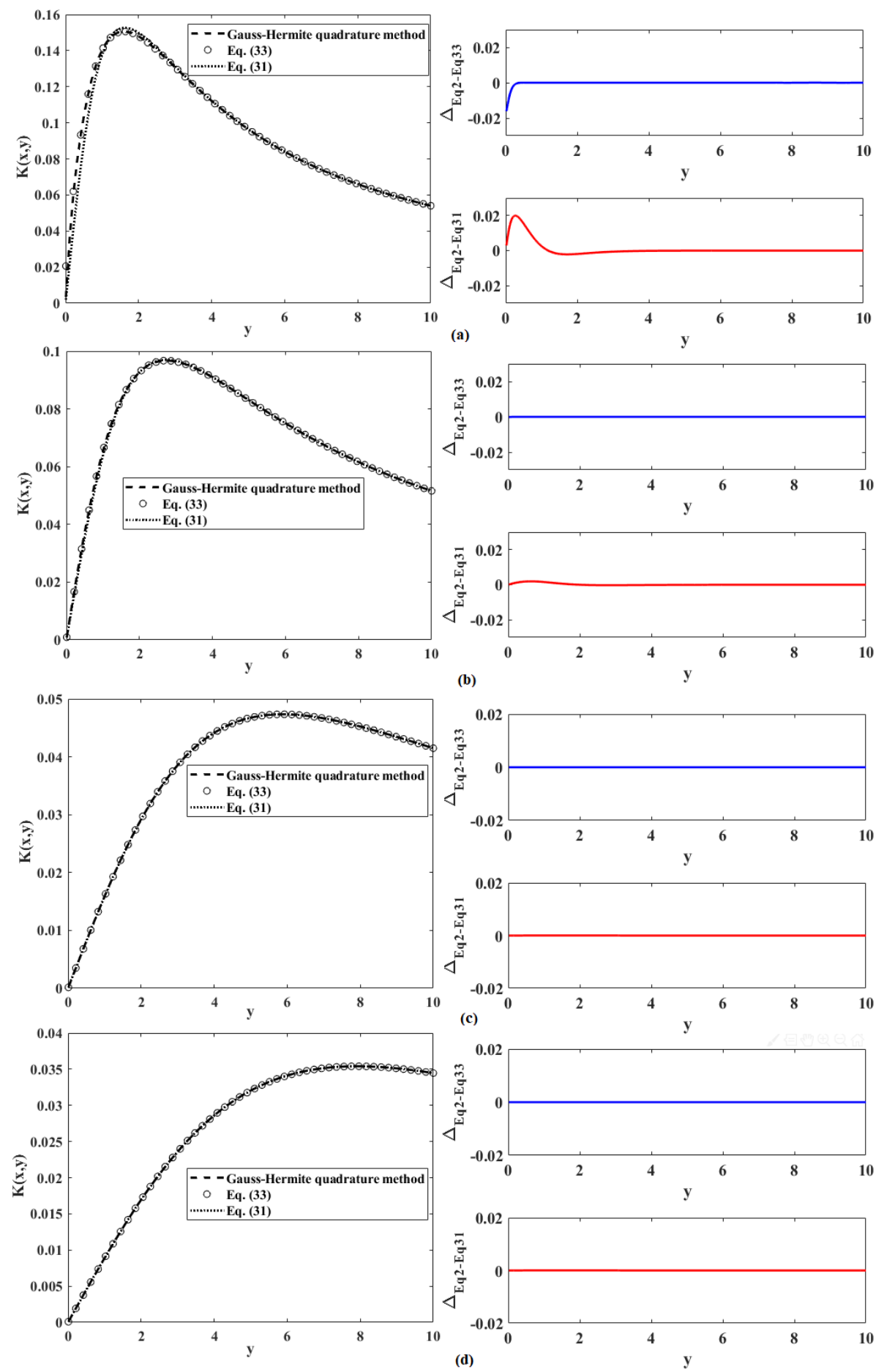

Figure 6. The Voigt function $K(x, y)$ in terms of $y$ for different values of $x$ : (a) $x=2,(\mathbf{b}) x=3$, (c) $x=6$, (d) $x=8$ and on the right side, the simulations of the difference between Equation (33), Equation (2) $\left(\Delta_{E q 2-E q 33}\right)$ and Equation (31), Equation (2) $\left(\Delta_{E q 2-E q 31}\right)$.

\section{Conclusions}

The Voigt profile is important place in several fields of physics. In the current investigation, an analytical formula, expressed in terms of a special function, is established. A simple approach is examined to facilitate the manipulation of the Voigt profile. To prove the validity of our new expression, some numerical examples are performed and discussed. The main goal of this work is to present to the readers a simple formula and rapid calculation. These benefits make the study of the Voigt profile more straightforward and 
convenient. The Voigt profile has an extensive range of applications in different domains such as astrophysics, spectroscopy, and the theory of neutron reactions, among others.

Author Contributions: Investigation, S.C.; writing-review and editing, A.B. All authors contributed equally to this work. All authors have read and agreed to the published version of the manuscript.

Funding: This research received no external funding.

Data Availability Statement: Not applicable.

Acknowledgments: We are grateful to members of our Laboratory for interesting discussions and useful comments. We are also grateful to the anonymous referees for their interesting constructive comments that helped improve the manuscript.

Conflicts of Interest: The authors declare no conflict of interest.

\section{References}

1. Reiche, F. Uber die emission, absorption and intensitats vertcitung von spektrallicien. Ber. Deutsch. Phys. Ges. 1913, 15, 3-21.

2. Benedict, W.S.; Herman, R.; Moore, G.E.; Silverman, S. The Strengths, Widths, and Shapes of Infrared Lines: I. General Considerations. Can. J. Phys. 1956, 34, 830-849. [CrossRef]

3. Hummer, D.G. Non-Coherent Scattering: I. The Redistribution Function with Doppler Broadening. Mon. Not. R. Astron. Soc. 1962, 125, 21-37. [CrossRef]

4. Armstrong, B. Spectrum line profiles: The Voigt function. J. Quant. Spectrosc. Radiat. Transf. 1967, 7, 61-88. [CrossRef]

5. Young, C. Calculation of the absorption coefficient for lines with combined Doppler and Lorentz broadening. J. Quant. Spectrosc. Radiat. Transf. 1965, 5, 549-552. [CrossRef]

6. Yamada, $\mathrm{H}$. Total radiances and equivalent widths of isolated lines with combined Doppler and collision broadened profiles. J. Quant. Spectrosc. Radiat. Transf. 1968, 8, 1463-1473. [CrossRef]

7. Haubold, H.J.; John, R.W. Spectral line profiles and neutron cross sections: New results concerning the analysis of Voigt functions. Astrophys. Space Sci. 1979, 65, 477-491. [CrossRef]

8. Whiting, E. An empirical approximation to the Voigt profile. J. Quant. Spectrosc. Radiat. Transf. 1968, 8, 1379-1384. [CrossRef]

9. Rodgers, C.; Williams, A. Integrated absorption of a spectral line with the Voigt profile. J. Quant. Spectrosc. Radiat. Transf. 1974, 14, 319-323. [CrossRef]

10. Drayson, S. Rapid computation of the Voigt profile. J. Quant. Spectrosc. Radiat. Transf. 1976, 16, 611-614. [CrossRef]

11. Minguzzi, P.; Di Lieto, A. Simple Padé approximations for the width of a Voigt profile. J. Mol. Spectrosc. 1985, 109, 388-394. [CrossRef]

12. Flores-Llamas, H.; Cabral-Prieto, A.; Jiménez-Domínguez, H.; Torres-Valderrama, M. An expression for an approximation of the Voigt profile I. Nucl. Instruments Methods Phys. Res. Sect. A Accel. Spectrometers Detect. Assoc. Equip. 1991, 300, 159-163. [CrossRef]

13. Teodorescu, C.; Esteva, J.; Karnatak, R.; El Afif, A. An approximation of the Voigt I profile for the fitting of experimental X-ray absorption data. Nucl. Instrum. Methods Phys. Res. Sect. A Accel. Spectrometers Detect. Assoc. Equip. 1994, 345, 141-147. [CrossRef]

14. Liu, Y.; Lin, J.; Huang, G.; Guo, Y.; Duan, C. Simple empirical analytical approximation to the Voigt profile. J. Opt. Soc. Am. B 2001, 18, 666-672. [CrossRef]

15. Asfaw, A. A fast method of modeling spectral lines. J. Quant. Spectrosc. Radiat. Transf. 2001, 70, 129-137. [CrossRef]

16. Letchworth, K.L.; Benner, D.C. Rapid and accurate calculation of the Voigt function. J. Quant. Spectrosc. Radiat. Transf. 2007, 107, 173-192. [CrossRef]

17. Di Rocco, H.; Cruzado, A. The Voigt Profile as a Sum of a Gaussian and a Lorentzian Functions, when the Weight Coefficient Depends Only on the Widths Ratio. Acta Phys. Pol. A 2012, 122, 666-669. [CrossRef]

18. Srivastava, H.M.; Miller, E.A. A unified presentation of the Voigt functions. Astrophys. Space Sci. 1987, 135, 111-118. [CrossRef]

19. Errera, Q.; Vanderauwera, J.; Belafhal, A.; Fayt, A. Absolute Intensities in 16O12C32S: The 2500-3100 cm ${ }^{-1}$. J. Mol. Spectrosc. 1995, 173, 347-369. [CrossRef]

20. Belafhal, A. The shape of spectral lines: Widths and equivalent widths of the Voigt profile. Opt. Commun. 2000, 177, 111-118. [CrossRef]

21. AlOmar, A.S. Accurate Chebyshev Approximations for the Width of the Voigt Profile, Differential Peaks, and Deconvolution of the Lorentzian Width. Optik 2021, 225, 165533. [CrossRef]

22. Watson, G.N. A Treatise on the Theory of Bessel Functions; Cambridge University Press: Cambridge, UK, 1995.

23. Gradshteyn, I.S.; Ryzhik, I.M.; Jeffrey, A. Table of Integrals, Series, and Products, 7th ed.; Academic Press: Amsterdam, The Netherlands; Boston, MA, USA, 2007.

24. Srivastava, H.M.; Karlsson, P.W. Multiple Gaussian Hypergeometric Series; Ellis Horwood: Chichester, UK, 1985.

25. Singh, D. On Some Representations of Voigt Functions. Ph.D. Thesis, Aligarh Muslim University, Aligarh, India, 2004.

26. Huang, Q.; Coëtmellec, S.; Duval, F.; Louis, A.; Leblond, H.; Brunel, M. Analytical expressions for diffraction-free beams through an opaque disk. J. Eur. Opt. Soc. Rapid Publ. 2011, 6, 11031-11037. [CrossRef]

27. Belafhal, A.; Chib, S.; Khannous, F.; Usman, T. Evaluation of integral transforms using special functions with applications to biological tissues. Comput. Appl. Math. 2021, 40, 1-23. [CrossRef] 